\title{
Theoretical Investigation of Indium Surface Segregation in InGaN Thin Films*
}

\author{
Shingo Inahama, ${ }^{\dagger}$ Toru Akiyama, Kohji Nakamura, and Tomonori Ito \\ Department of Physics Engineering, Mie University, \\ Kurima Machiya 157\%, Tsu, Mie 514-8507, Japan \\ (Received 14 October 2005; Accepted 6 December 2005; Published 27 December 2005)
}

\begin{abstract}
Surface segregation of In atoms in InGaN thin films on GaN(0001) substrate is investigated by using Monte Carlo simulations based on an empirical potential, which incorporates electrostatic energy due to bond charge and ionic charges. The calculated In composition of the surface monolayer (ML) $x_{s}$ in $\mathrm{In}_{0.1} \mathrm{Ga}_{0.9} \mathrm{~N}$ thin films ranging from 1 to $31 \mathrm{ML}$ predicts that In atoms segregate at the topmost layer even in the film thickness $t$ being larger than $3 \mathrm{ML}$. The $x_{s}$ at the topmost layer saturates when $t$ reaches $\sim 15 \mathrm{ML}$, in which $x_{s}$ is much larger $\left(x_{s} \sim 0.7\right)$ than the normal alloy composition $(x \sim 0.1)$. Furthermore, analysis of $x_{s}$ with respect to the bulk composition $x$ up to 0.2 reveals that the propensity of In atoms for being segregated at the surface corresponds to the bond energy difference between InN and GaN. These calculated results imply that not only the release of elastic strains due to lattice mismatch between InN and GaN but also the preference of Ga-N bonds over In-N bonds in the bulk region contributes the segregation of In atoms at the surface in InGaN thin films. [DOI: 10.1380/ejssnt.2005.503]
\end{abstract}

Keywords: InGaN; surface segregation; Monte Carlo simulation; empirical interatomic potential; thin films

\section{INTRODUCTION}

GaN based III-V compound semiconductors are attractive materials for their promises in optoelectronic application. In particular, InGaN has been paid much attention in recent years for their application such for light emitting diodes covered continuously from the ultra-violet to infrared region by proper allying. However, the fabrication of compositionally controlled InGaN films has been shown to be difficult, because atomic size between In and Ga atoms is quite different with each other, and thermal expansion coefficient and mechanical properties of $\mathrm{InN}$ are different from those of GaN. For instance, it has been reported that In surface segregation effect, in which In atoms segregate along the growth axis, occurs during the growth of InGaN ternary alloys [1, 2] and influences on the composition profiles in heterostructures [3]. Despite the importance of clarifying microscopic origins in In surface segregation in InGaN thin films, however, there have been very few experimental studies from atomistic viewpoints although detailed analysis of In surface segregation in other ternary alloys such as InGaAs and AlGaAs have been carried out $[4,5]$. Furthermore, theoretical investigations for this purpose have been rarely carried out at present.

In our previous study, thermodynamic stabilities of InGaN films have been investigated based on an empirical interatomic potential calculation $[6,7]$, and then our empirical potential [6-12] is found to be feasible for investigating the structural stability for GaN-based alloys. In this study, In surface segregation of InGaN thin films on $\mathrm{GaN}(001)$ substrates are investigated by using our empirical potential. Monte Carlo (MC) simulations based on our empirical potential are performed to determine the preferable atomic configurations of cation atoms for various film thickness and cation composition.

\footnotetext{
* This paper was presented at International Symposium on Surface Science and Nanotechnology (ISSS-4), Saitama, Japan, 14-17 November, 2005.

†Corresponding author: inahama03@nd.phen.mie-u.ac.jp
}

\section{COMPUTATIONAL METHODS}

Surface segregation of In atoms in InGaN thin films is investigated by the MC simulation. Here, we consider the system consisting of $\mathrm{GaN}(0001)$ substrate with 9 layers and pseudomorphically grown InGaN thin films ranging from 1 to 31 monolayer (ML). A $(10 \times 10)$ surface unit cell is used in this study. The lattice parameter of basal plane $a$ is fixed to be that of $\mathrm{GaN}(0001)$ substrate, and the lattice parameter parallel to the growth direction $c$ and displacement of atoms are varied to minimize the system energy. We consider $\operatorname{InGaN}(0001)-(1 \times 1)$ ideal surface as a representative of surface structures because the energy difference between the $(1 \times 1)$ structure and other structure such as the $(2 \times 2)$ structure is found to be smaller than the cohesive energy of InGaN. In the calculation procedure, randomly chosen atoms in the thin films layers are replaced and all atoms in the system are displaced to equilibrate the system according to the Metropolis MC algorithm. Equilibrium atomic arrangements are obtained at the temperature of $520^{\circ} \mathrm{C}$, which corresponds to the experimentally reported temperature for InGaN crystal growth and also coincide with the substrate temperature for In surface segregation in InGaAs thin films [4]. Interdiffusion between thin film and substrate layers is neglected in this study. The content of In atoms in $\operatorname{In}_{x} \mathrm{Ga}_{1-x} \mathrm{~N}$ thin films considered in this study is $0<x<0.2$, where the promising range of $x$ as no phase separation is experimentally confirmed [13]. Furthermore, the temperature dependence on In surface segregation is investigated from the $\mathrm{MC}$ simulations for $\mathrm{In}_{0.1} \mathrm{Ga}_{0.9} \mathrm{~N}$ at $520^{\circ} \mathrm{C}$ and $800^{\circ} \mathrm{C}$.

The system energy used in the MC simulation is obtained by using our empirical interatomic potential expressed as $[6-8,14,15]$ :

$$
\begin{aligned}
E & =E_{0}+E_{e s}, \\
E_{0} & =\frac{1}{2} \sum_{i, j} V_{i j}, \\
E_{e s} & =K\left[\frac{3}{2}\left(1-f_{i}\right) \frac{Z_{b}^{2}}{r_{b b}}-f_{i} \frac{Z_{i}^{2}}{r_{i i}}\right] .
\end{aligned}
$$

Here, $E_{0}$ is the cohesive energy estimated by Kohr-Das 


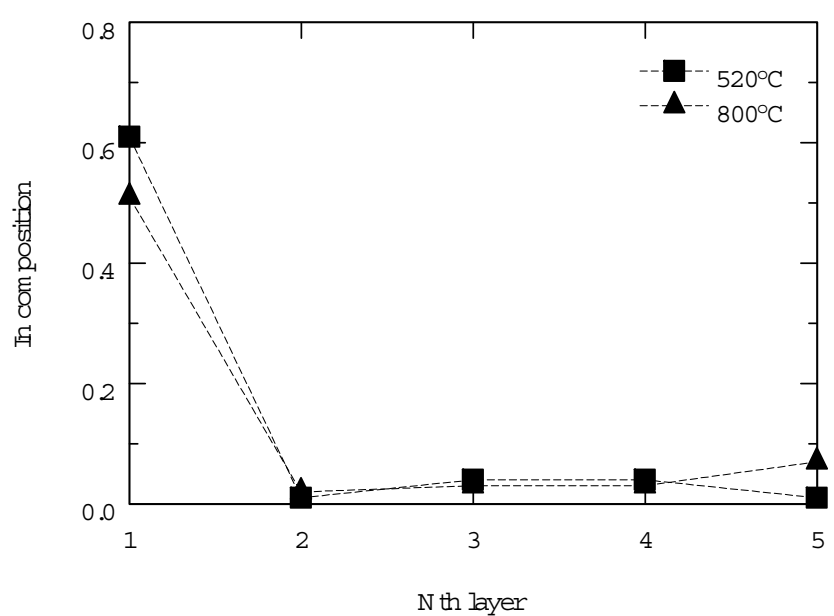

FIG. 1: In composition profile $x_{s}$ near surface in $\operatorname{In}_{0.1} \mathrm{Ga}_{0.9} \mathrm{~N}$ thin films with 15 ML thickness on GaN substrates. Squares and triangles correspond to $x_{s}$ at $520^{\circ} \mathrm{C}$ and $800^{\circ} \mathrm{C}$, respectively.

Sarma type empirical interatomic potential within the second neighbor interactions [16, 17], and $E_{e s}$ is the electrostatic interaction between covalent bond charges $\left(Z_{b}=2\right)$ and that between ionic charges $\left(Z_{i}=3\right.$ for III$\mathrm{V}$ semiconductors) beyond the second nearest neighbors depending on ionicity $f_{i}$, which corresponds to the firstneighbor interlayer interaction $[14,15] . r_{b b}$ and $r_{i i}$ are the distance between covalent bond charges and that between ionic charges, respectively. The value of coefficient $K$ used throughout this study is $8.7 \mathrm{meV} \cdot \AA[18]$. Using Eq. (1), the system energy is calculated using the above-mentioned unit cell consisting of $\sim 4000$ atoms for the hypothetical InGaN thin films on GaN(0001) substrate.

\section{RESULTS AND DISCUSSION}

Our MC simulations for $\operatorname{In}_{0.1} \mathrm{Ga}_{0.9} \mathrm{~N}$ thin films ranging from 1 to 31 ML show that In composition in the surface ML $x_{s}$, the ratio of In atoms to the total number of cation atoms in each layer, takes large values at the topmost layer and varies rapidly in the second ML being small values compared with the bulk composition 0.1 . This trend is clearly seen when the thickness $t$ is larger than $3 \mathrm{ML}$. Figure 1 shows $x_{s}$ in $\mathrm{In}_{0.1} \mathrm{Ga}_{0.9} \mathrm{~N}$ thin films of $15 \mathrm{ML}$ as a function of cation-atom-layer at $520^{\circ} \mathrm{C}$ and $800^{\circ} \mathrm{C}$, as a typical case of our MC simulations: $x_{s}$ is 0.6 at the topmost layer and is smaller than the bulk composition 0.1 for the other layers. It is thus shown that In atoms are segregated at the topmost layer. Our analysis of the system energy shows that this characteristic can be interpreted as the bond energy difference between In-N and Ga-N bonds. Since the energy of In-N bond (1.86 $\mathrm{eV}$ ) in the bulk phase is lower than that of $\mathrm{Ga}-\mathrm{N}$ bond $(2.34 \mathrm{eV})$, the energy loss by taking three-coordinated In atoms at the topmost layer $(0.98 \mathrm{eV}))$ is lower than that by taking three-coordinated Ga atom $(1.12 \mathrm{eV})$, leading to the preference of In atoms at the topmost layer. We also find that the value of $c / a$ for the segregated films (1.660) is found to be large compared with that for the

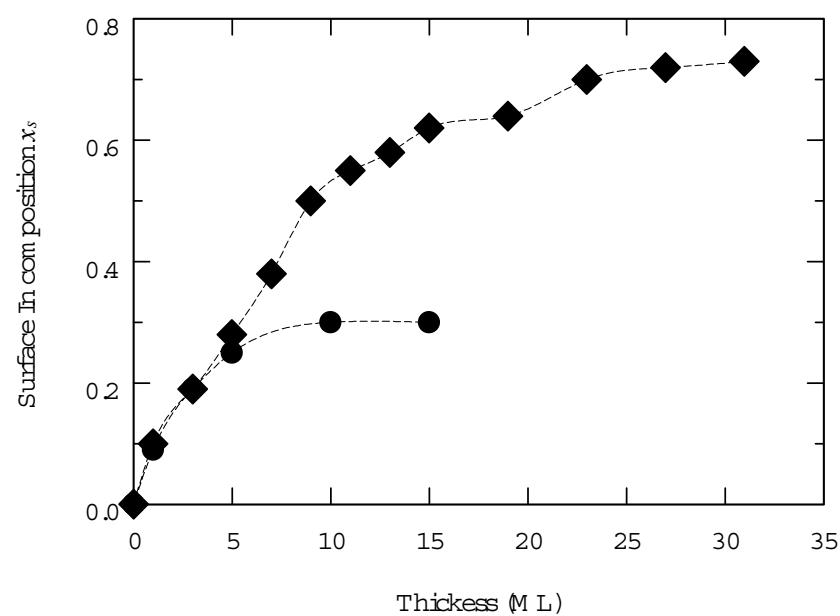

FIG. 2: Surface In composition $x_{s}$ at the topmost layer of $\mathrm{In}_{0.1} \mathrm{Ga}_{0.9} \mathrm{~N}$ thin films (squares) as a function of its layer thickness $t$ at $520^{\circ} \mathrm{C}$. Experimentally reported surface In composition $x_{s}$ of $\operatorname{In}_{0.1} \mathrm{Ga}_{0.9} \mathrm{As}$ (circles) is also shown.

InGaN film in which In atoms are randomly distributed (1.633), implying that elastic strains due to the difference between InN and GaN still remain in the lateral direction. Therefore, the energy gain by reducing In composition in the bulk-like region also contribute In surface segregation. Furthermore, our MC simulation at $800^{\circ} \mathrm{C}$, until which the InGaN film growth is successfully demonstrated [19], shows that $x_{s}$ at the topmost layer is slightly lower than that at $520^{\circ} \mathrm{C}$. However, it takes much large value compared with other layers, indicating that In atoms are segregated at this temperature. This result implies that In surface segregation occurs over the entire temperature range in InGaN film growth.

Figure 2 shows $x_{s}$ at the topmost layer as a function of film thickness of $\operatorname{In}_{0.1} \mathrm{Ga}_{0.9} \mathrm{~N}$. As $t$ increases up to $\sim 15 \mathrm{ML}, x_{s}$ at the topmost layer monotonically increases. Since most of In atoms are located at the topmost layer in this range, $x_{s}$ is approximately proportional to film thickness. $x_{s}$ at the topmost layer saturates when $t$ reaches more than $15 \mathrm{ML}$, in which $x_{s}(\sim 0.7)$ is much larger than the normal alloy composition $x=0.1$. This trend is qualitatively consistent with the experimental results of InGaAs thin films, although the saturated value of $x_{s}$ at the topmost layer of $\operatorname{In}_{0.1} \mathrm{Ga}_{0.9} \mathrm{~N}$ is different from that of $\operatorname{In}_{0.08} \mathrm{Ga}_{0.92} \mathrm{As}$ [4]. The saturated value of $x_{s}$ at the topmost layer indicates that $x_{s}$ of the topmost layer is in equilibrium with a bulk layer of composition. Our analysis of the saturated $x_{s}$ at the topmost layer clarifies that the equilibrium condition can be quantified in term of the energy gain for In surface segregation: The $x_{s}$ at the topmost layer agrees with the McLean's-type equation [20]:

$$
\ln \left[\frac{x_{s}}{1-x_{s}}\right]+\frac{E_{s}}{k_{B} T}=\ln \left[\frac{x}{1-x}\right],
$$

where the parameter $E_{s}$ describes the propensity of In atoms for being segregated at the surface, including the contribution of both bond energy and strain related effects. The $E_{s}=0.18 \mathrm{eV}$ obtained from $\left(x_{s}, x\right)=(0.7$, 


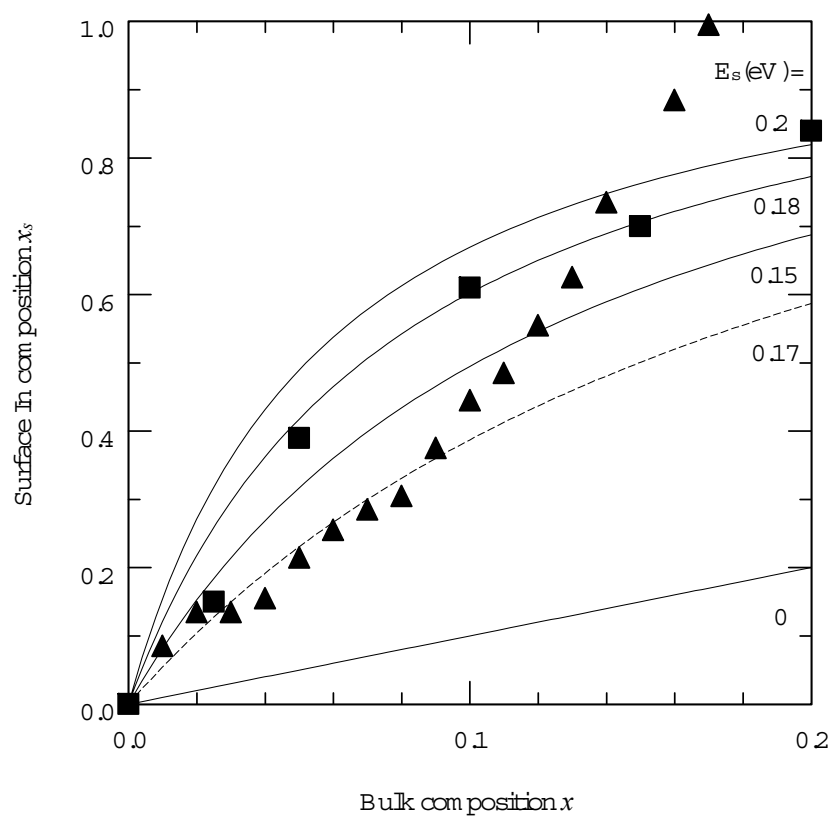

FIG. 3: Surface In composition $x_{s}$ at the topmost layer of $\mathrm{In}_{x} \mathrm{Ga}_{1-x} \mathrm{~N}$ films (squares) with $15 \mathrm{ML}$ thickness at $520^{\circ} \mathrm{C}$ as a function of bulk In composition $x$. Triangles represents $x_{s}$ of $\mathrm{In}_{x} \mathrm{Ga}_{1-x}$ As obtained by experiments. Solid and dashed lines indicate $x_{s}$ at the topmost layer obtained from Eq. (4) using various values of $E_{s}$ in InGaN and InGaAs, respectively.

0.1) reasonably agrees with the energy difference threecoordinated In and $\mathrm{Ga}$ atoms $(0.14 \mathrm{eV})$. This implies that In atoms preferably reside in the surface lattice sites compared to Ga atoms. We conjecture that the slight difference between Es and the energy difference between threecoordinated In and $\mathrm{Ga}$ atoms is due to the effects of elastic strains in the bulk region.

The relation between $x_{s}$ at the topmost layer and bulk composition $x$ expressed in Eq. (4) holds in a wide range of bulk composition. Figure 3 shows $x_{s}$ at the topmost layer of $\mathrm{In}_{0.1} \mathrm{Ga}_{0.9} \mathrm{~N}$ with $t=15 \mathrm{ML}$, which is considered to be sufficiently large for the surface composition $x_{s}$ layer to be in saturation regime, as a function of bulk composition $x$. The values obtained from Eq. (4) for various $E_{s}$ are also shown. The $\left(x_{s}, x\right)$ plots obtained from our MC simulations agrees well with those from Eq. (4) using $E_{s}=0.18 \mathrm{eV}$, indicating that the energy gain for In atoms being located at the surface is the principle factor. $\left(x_{s}, x\right)$ for low $x$ is qualitatively consistent with experimental results of InGaAs thin films. However, a large deviation from the experimental value is recognized for large In composition $(x>0.1)$. Since the experimental data imply the enhancement of In surface roughening [4] and dislocations formation [5], these effects might also enhance the In surface segregation in $\operatorname{In}_{x} \mathrm{Ga}_{1-x} \mathrm{~N}$ films, especially for large $x$. Although clarifying these effects, which is left out of this study, should be carried out in subsequent calculations, our approach using MC calculations give the correct qualitative trends for surface segregation of semiconductor alloy thin films.

\section{CONCLUSIONS}

We have investigated In surface segregation in InGaN thin films on GaN(0001) substrate by using Monte Carlo simulations based on an empirical potential. We have found that In atoms segregate at the topmost layer in the film thickness $t$ being larger than $3 \mathrm{ML}$. This is because the energy loss taking three-coordinated In atom at the topmost layer is lower than that by taking threecoordinated $\mathrm{Ga}$ atom. We have also found that $x_{s}$ in $\mathrm{In}_{0.1} \mathrm{Ga}_{0.9} \mathrm{~N}$ saturates when $t$ reaches more than $15 \mathrm{ML}$ in which the $x_{s}$ is much larger $\left(x_{s} \sim 0.7\right)$ than the normal alloy composition $\left(x_{s} \sim 0.1\right)$, qualitatively consistent with the experimental findings in InGaAs thin films. Analysis of $x_{s}$ with respect to the bulk composition $x$ also reveals that the propensity of In atoms for being segregated at the surface corresponds to the bond energy difference between InN and GaN. Therefore, these calculated results imply that not only the release of the elastic strain due to lattice mismatch between $\mathrm{InN}$ and $\mathrm{GaN}$ but also the preference of In atoms at the topmost layer contributes the segregation of In atoms at the surface.

\section{Acknowledgments}

Computations were partly done at RCCS (National Institutes of Natural Sciences) and ISSP (University of Tokyo).
[1] C. Kisielowski, Z. Liliental-Weber, and S. Nakamura, Jpn. J. Appl. Phys. 36, 6932 (1997).

[2] N. Duxbury, U. Bangert, and P. Dawson, Appl. Phys. Lett. 76, 1600 (2000).

[3] R. Shingh, D. Doppalapudi, T. D. Moustakes, and, L. T. Tomano, Appl. Phys. Lett. 70, 1089 (1997).

[4] J. M. Gerard, Appl. Phys. Lett. 61, 2069 (1992).

[5] T. Kawai, H. Yonezu, Y. Ogasawara, D. Saito, and K. Pak, J. Appl. Phys. 74, 1770 (1993).

[6] Y. Kangawa, T. Ito, A. Mori, and A. Koukitu, J. Cryst. Growth 220, 401 (2000).

[7] Y. Kangawa, T. Ito, Y. Kumagai, A. Koukitu, and N. Kawaguchi, Jpn. J. Appl. Phys. 42, L95 (2003).
[8] T. Ito, and Y. Kangawa, J. Cryst. Growth 235, 149 (2002).

[9] T. Suda, Y. Kangawa, K. Nakamura, and T. Ito, J. Cryst. Growth 258, 277 (2003).

[10] T. Ito, T. Suda, T. Akiyama, and K, Nakamura, Appl. Surf. Sci. 244, 170 (2005).

[11] K. Kawamoto, T. Suda, T. Akiyama, K. Nakamura, and T. Ito, Appl. Surf. Sci. 244, 182 (2005).

[12] T. Ito, K. Sano, T. Akiyama, and K. Nakamura, Thin Solid Films, in press (2005).

[13] T, Miyajima, Y. Kudo, K. -Y. Liu, T. Uruga, T. Asatsuma, T. Hino, and T. Kobayashi, Phys. Stat. Sol. (b) 228, 45 (2001). 
[14] T. Ito, Jpn. J. Appl. Phys. 37, 1217 (1998).

[15] T. Ito, K. Shiraishi, and A. Taguchi, J. Cryst. Growth $\mathbf{2 2 7} / \mathbf{2 2 8}, 336$ (2001).

[16] T. Ito, K. E. Khor, and A. Das Sarma, Phys. Rev. B 41, 3893 (1990).

[17] T. Ito, J. Appl. Phys. 77, 4947 (1995).

[18] C. -Y. Yeh, Z. W. Lu, S. Froyen, and A. Zunger, Phys.
Rev. B 46, 10086 (1992)

[19] L. W. Wu, S. J. Chang, Y. K. Su, R. W. Chuang, Y. P. Hsu, C. H. Kuo, W. C. Lai, T. C. Wen, J. M. Tsai, and J. K. Sheu, Solid-State Electronics 47, 2027 (2003).

[20] G. A. Chadwick and D. A. Smith, Grain Boundary Structure and Properties, (Academic, London, 1975). 